\title{
Neonatal Marfan Syndrome: Report of a Case with an Inherited Splicing Mutation outside the Neonatal Domain
}

\author{
Laurianne Le Gloan ${ }^{\mathrm{a}}$ Quentin Hauet ${ }^{\mathrm{a}}$ Albert David $^{\mathrm{b}}$ Nadine Hanna $^{\mathrm{d}}$
}

Chloé Arfeuille $^{d}$ Pauline Arnaud $^{d}$ Catherine Boileau $^{d}$ Bénédicte Romefort ${ }^{\mathrm{a}}$

Nadir Benbrik $^{\mathrm{a}}$ Véronique Gournay ${ }^{\mathrm{a}}$ Nicolas Joram ${ }^{c}$ Olivier Baron ${ }^{\mathrm{a}}$

Bertrand Isidor ${ }^{b}$

${ }^{a}$ Cardiologie Pédiatrique et Congénitale, ${ }^{b}$ Génétique Médicale, and ${ }^{c}$ Réanimation Pédiatrique, $\mathrm{CHU}$ de Nantes, Université de Nantes, Nantes, and d Département de Génétique Moléculaire, Centre National de Référence pour le Syndrome de Marfan et Apparentés, INSERM LVTS U1148, Faculté Paris Diderot, AP-HP Hopital Bichat, Paris, France

\section{Established Facts}

- Neonatal presentations of Marfan syndrome are usually subsequent to de novo mutations located in the so-called neonatal domain of the FBN1 gene, in the region of exons 24-32.

\section{Novel Insights}

- We report a single, so far undescribed mutation in the FBN1 gene, located outside the neonatal domain, and responsible for familial Marfan syndrome, involving a mother, with a common clinical presentation, and her child, with a neonatal presentation. No argument for mosaicism was found.

\section{Key Words}

FBN1 · Fibrillin · Marfan syndrome · Neonatal Marfan syndrome

\footnotetext{
Abstract

We report a child and her mother affected by Marfan syndrome. The child presented with a phenotype of neonatal Marfan syndrome, revealed by acute and refractory heart failure, finally leading to death within the first 4 months of
}

life. Her mother had a common clinical presentation. Genetic analysis revealed an inherited FBN1 mutation. This intronic mutation (c.6163+3_6163+6del), undescribed to date, leads to exon 49 skipping, corresponding to in-frame deletion of 42 amino acids (p.Ile2014_Asp2055del). FBN1 nextgeneration sequencing did not show any argument for mosaicism. Association in the same family of severe neonatal and classical Marfan syndrome illustrates the intrafamilial phenotype variability.

(c) 2016 S. Karger AG, Basel

\section{KARGER 125}

2016 S. Karger AG, Basel

$1661-8769 / 16 / 0066-0281 \$ 39.50 / 0$

E-Mail karger@karger.com

www.karger.com/msy
Laurianne Le Gloan

Cardiologie, Institut du Thorax, CHU de Nantes

Boulevard Jacques Monod

FR-44093 Nantes Cedex 01 (France)

E-Mail laurianne.legloan@ chu-nantes.fr 
Fig. 1. Photographs of the child's fingers (a) and toes (b) revealing marked arachnodactyly.
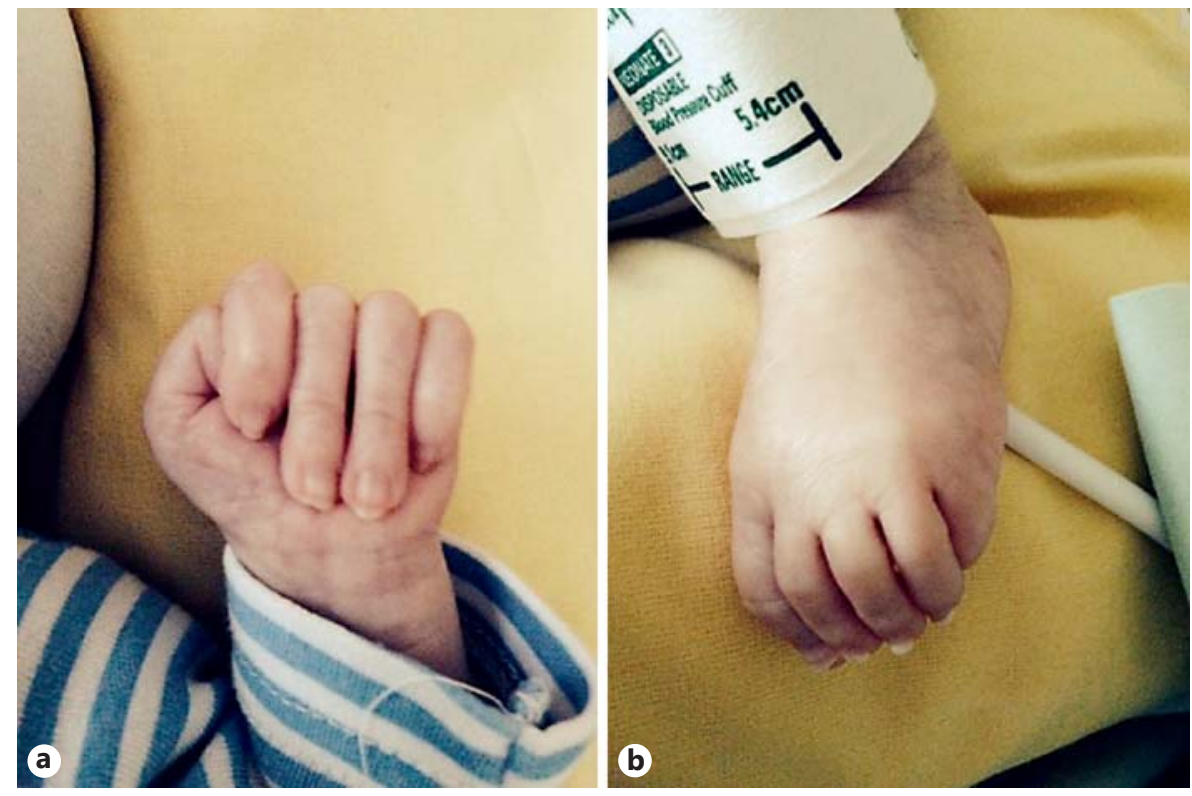

Marfan syndrome is an inherited disorder of the connective tissue responsible for heterogeneous phenotypic presentation [Bolar et al., 2012]. Neonatal presentations are severe, progressive, with a mortality rate close to $50 \%$ due to cardiovascular complications [Faivre et al., 2009b]. They mostly occur as de novo mutations, located in the region of exons 24-32 of the FBN1 gene. Here, we report a child and her mother affected by Marfan syndrome caused by a novel FBN1 gene mutation localized outside the neonatal domain and presenting, respectively, with a severe neonatal and a classical form.

\section{Case Report}

\section{Patient 1}

The proband was a child born after 39 weeks of gestation. Obstetrical and fetal follow-up had revealed no anomalies. Because of a fetal breech position, a caesarean section was performed at term. Height, weight and OFC were $50 \mathrm{~cm}$ (0 SD), 2,950 g (-0.5 SD) and $33.5 \mathrm{~cm}$ ( $-1 \mathrm{SD})$, respectively. At birth, the girl immediately suffered from acute respiratory distress, related to heart failure. Clinical examination revealed arachnodactyly (fig. 1), facial dysmorphism with senile appearance and loose skin. Transthoracic echocardiography revealed severe mitral and tricuspid valvular regurgitations, moderate aortic root dilatation $(16 \mathrm{~mm}, \mathrm{Z}$ score 5 ; fig. 2) with moderate aortic regurgitation. High doses of diuretics and mechanical ventilation were rapidly required with transient relief of the symptoms. The initial course was marked by the occurrence of a diaphragmatic hernia at 4 weeks of life, successfully treated by surgical correction. Despite optimized medical therapy, heart failure was complicated by failure to thrive at 2 months of age, requiring surgical mitral and tricuspid valve annuloplasties with partial success. After transient improvement allowing weaning from mechanical ventilation, further respiratory deterioration occurred, favored by spontaneous pneumothorax. The child finally died at 4 months of age of a cardiac arrest secondary to severe hypoxemia. Anthropometric features, particularly height, were within normal ranges, although numeric data were not available.

\section{Patient 2}

The child's mother is a 32-year-old woman with clinically diagnosed Marfan syndrome. She had been adopted during childhood; therefore, no familial history was available. The child's father had no relevant past medical history. The mother had been followed-up since childhood for surgically corrected ectopia lentis and aortic root dilatation without any other systemic features of Marfan syndrome. She was on $\beta$-blocker therapy (atenolol $100 \mathrm{mg}$ per day), with scarce cardiac follow-up, and made no prior attempt to obtain molecular confirmation of the diagnosis. Cardiac followup throughout pregnancy and 6 months after was uneventful with aortic root diameters remaining stable at $42 \mathrm{~mm}$ (fig. 3), under the same doses of atenolol. Genetic counseling was offered to the parents during pregnancy, and they declined further explorations as no prenatal diagnosis would have changed their decision to continue pregnancy.

\section{Results}

Total genomic DNA was isolated from blood lymphocytes from the child and her mother after informed consent was obtained from the parents. Sanger FBN1 gene sequencing analysis revealed a novel previously unpublished intronic mutation, the deletion of 4 nucleotides on 
Fig. 2. The child's transthoracic echocardiography, performed within the first days of life. Parasternal long-axis view showing aortic root dilatation at the level of the sinuses of Valsalva (15.9 mm, Z score 5.5). $\mathrm{LV}=$ Left ventricle; $\mathrm{LA}=$ left atrium; $\mathrm{AoR}=$ aortic root; $\mathrm{RV}=$ right ventricle.

Fig. 3. The mother's transthoracic echocardiography performed after delivery. Parasternal long-axis view showing moderate aortic root dilatation, at the level of the sinuses of Valsalva (42.3 mm, Z score 4.6). For abbreviations, see figure 2 .
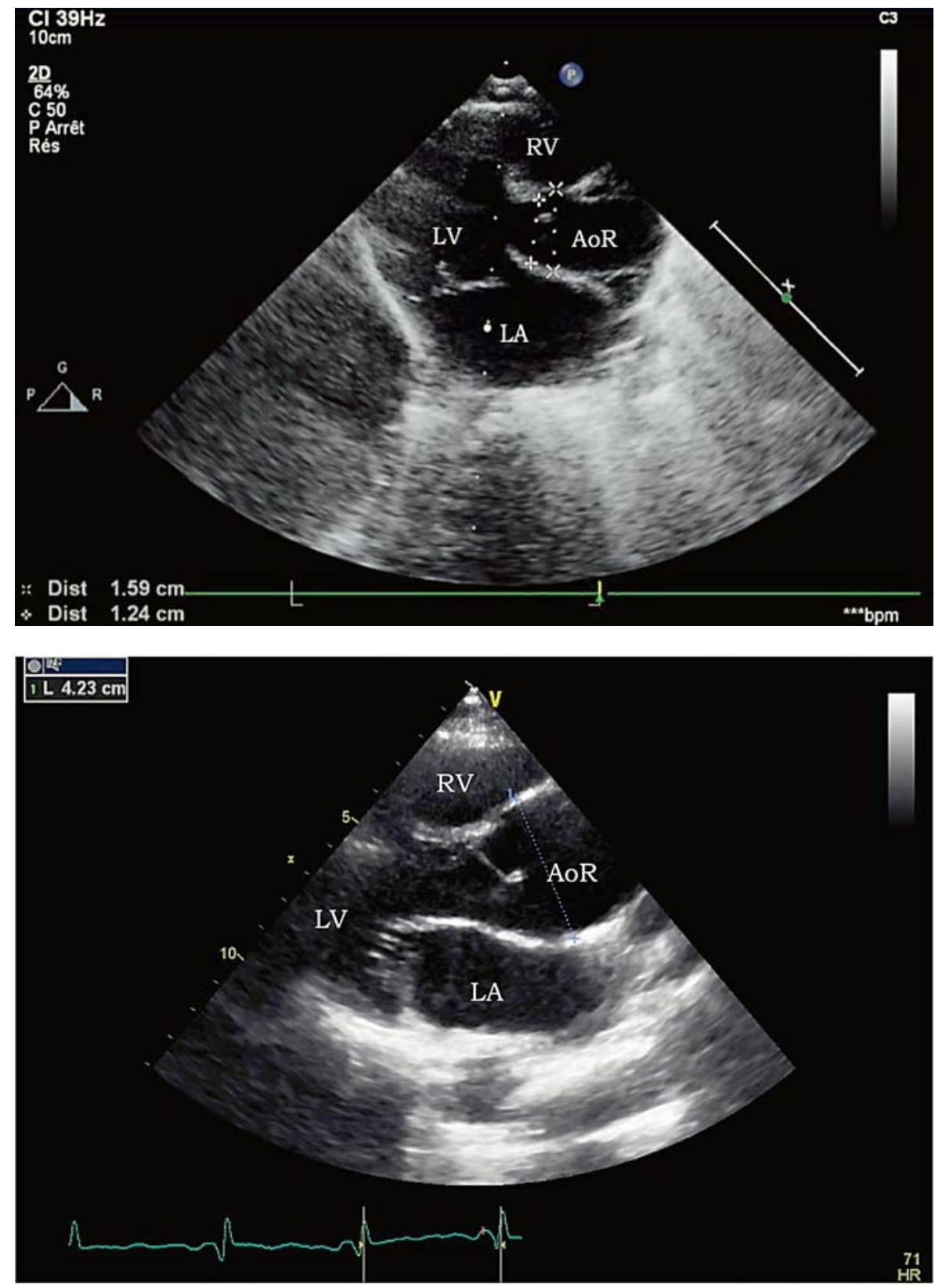

positions 3-6 upstream of exon 49 (c.6163+3_6163+6del) in a heterozygous state.

Total RNA of the mother was then isolated from fresh blood samples collected on PAXgene blood RNA tube (Qiagen S.A.S., France) and subjected to random hexamer-primed RT-PCR. The obtained cDNA products were amplified by PCR with exonic primers specific for the fragment of interest (primers and PCR details are available upon request). All cDNA fragments were separated according to size using acrylamid gel electrophoresis along with a DNA ladder. The amplification of the region including exons 46-50 of the mother's blood-derived cDNA was expected to reveal a single 617-bp fragment (wild-type allele) but showed the appearance of 2 bands (corresponding to wild type and the mutant; fig. 4). Direct sequencing of the amplified region revealed exon 49 skipping of the FBN1 transcript in the patient's mother (r.6038_6163del). The resulting transcript is in frame, leading to the deletion of 42 amino acids (p.Ile2014_Asp2055del). 


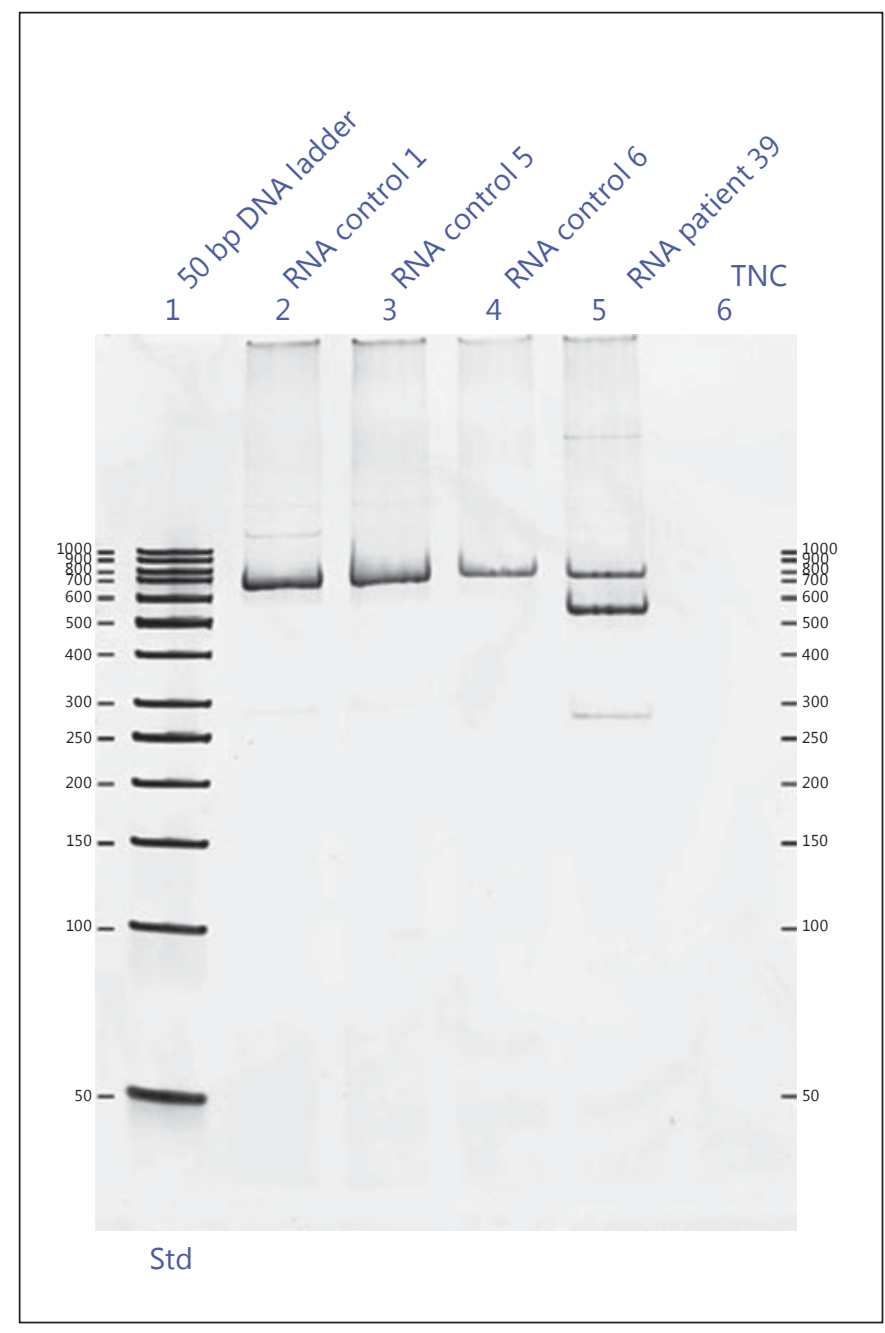

Fig. 4. RT-PCR results demonstrating the presence of one fragment of 617 bp in 3 normal controls (controls 1, 5 and 6) and 2 fragments (617 bp and abnormal $491 \mathrm{bp}$ ) in the blood-derived cDNA from the patient's mother (RNA patient 39 ). TNC $=$ Template negative control; Std $=$ Standard.

Sanger sequencing of the mother's mutation did not suggest mosaicism, since electropherograms obtained demonstrate a completely identical profile between herself and her daughter (online suppl. fig. 1; see www. karger.com/doi/10.1159/000443867). To further explore the possibility of a mosaic mutation, FBN1 next-generation sequencing (NGS) covering 65 exons of the gene and $50 \mathrm{bp}$ upstream and downstream of each exon was further performed (library preparation by TruSeq Custom Amplicon technology, Illumina ${ }^{\circledR}$, sequencer MiSeq, Illumina): the mutation was identified in $56 \%$ reads $(605 / 1,076)$ in the mother and $47 \%$ reads $(497 / 1,051)$ in the proband.

\section{Discussion}

The association of clinical features suggestive of Marfan disease (facial dysmorphism and arachnodactyly), refractory heart failure attributable to severe valvular regurgitations and spontaneous pneumothorax are typical of neonatal Marfan syndrome. The genetic analysis revealed the variant (c.6163+3_6163+6del) in the intron 49 of the $F B N 1$ gene in our proband inherited from her mother. $\mathrm{We}$, therefore, report a neonatal Marfan syndrome inherited from a mother presenting with classical Marfan syndrome, related to a novel previously unpublished mutation located outside the so-called neonatal domain region of exons 24-32. This observation may raise several genetic hypotheses.

Mutations in the gene encoding fibrillin-1, FBN1, are known to be responsible for the disease and are widely distributed along the entire gene. Nonetheless, genotypephenotype correlation is not sufficient enough to accurately predict the clinical manifestation in a given affected patient as demonstrated in our family. Mutations which spread through exons 24-32, particularly in-frame ones, are believed to play a significant role in neonatal forms [Putnam et al., 1996; Booms et al., 1999; Tiecke et al., 2001; Faivre et al., 2009a], although not exclusively, as shown in our family.

Most cases of Marfan syndrome with a neonatal presentation reported so far were due to de novo mutations. Nonetheless, several reports have pointed out the possible familial transmission of an FBN1 mutation resulting in a neonatal presentation. Recently, a child born with features of neonatal Marfan syndrome was reported with a heterozygous c. $3959 \mathrm{G}>\mathrm{A}$ transition mutation in exon 31 of the FBN1 gene, inherited from his father, and was also affected by Marfan syndrome [Elshershari and Harris, 2013]. A mosaic mutation in the child's mother might have explained her milder phenotype, but FBN1 NGS did not support this hypothesis.

In 2014, a novel intronic mutation in the FBN1 gene leading to exon 35 skipping was reported in a child affected by neonatal Marfan syndrome. Interestingly, although the family history of Marfan syndrome was negative, somatic mosaicism was identified in maternal blood cells (10-25\% of genomic DNA), and detailed clinical examination showed unilateral lens ectopy [Sipek et al., 2014]. FBN1 somatic or germline mutation mosaicism is a rare, yet well-documented phenomenon [Montgomery et al., 1998; Collod-Béroud et al., 1999; Rantamäki et al., 1999; Tekin et al., 2007; Hilhorst-Hofstee et al., 2011] and possibly underestimated. 
A case of neonatal presentation of Marfan syndrome has been previously attributed to a double mutant allele in exon 26 of FBN1 [Wang et al., 1996]. Although it has not been identified by Sanger and NGS on the FBN1 gene, we cannot rule out the presence of a second molecular event to explain the severe clinical presentation of our proband.

Marfan syndrome is notable for its variability regarding age of onset, tissue distribution, and severity of clinical manifestations. The neonatal presentation is recognized as the most severe end of the phenotypic spectrum. Only about $14 \%$ of all children with Marfan syndrome become apparent during the neonatal period [Faivre et al., 2009b]. Some authors propose to reserve this terminology to patients diagnosed at birth or within the first 3 months of life, presenting with pronounced atrioventricular valve dysfunction, frequently complicated by fatal congestive heart failure within the first year of life [Morse et al., 1990; Booms et al., 1999; Sutherell et al., 2007]. Pulmonary emphysema, severe valvular regurgitations, and loose skin are often associated [Hennekam, 2005]. Life expectancy seems to be the central issue when managing a patient with early diagnosed Marfan syndrome with cardiovascular involvement. Some prognostic factors associated with early death have been pointed out from a retrospective cohort of 60 probands carrying mutations in the FBN1 gene and with cardiovascular involvement before 1 year of age [Stheneur et al., 2011]. Factors significantly associated with shorter survival are presence of valvular insufficiencies, diaphragmatic hernia and a mutation in exons 25 or 26 [Stheneur et al., 2011]. However, several cases of neonatal Marfan syndromes with favorable outcome, up to 9 years after cardiac surgical management, have nonetheless been reported, but their initial presentations were less severe than our case [Rozendaal et al., 2011; Brito-Filho et al., 2013]. Furthermore, some prenatal findings may have been described in the literature, particularly cardiomegaly and aortic and pulmonary trunks enlargement [Gavilán et al., 2011]. Cardiac fetal echocardiography is still not recommended in international guidelines for pregnant women with Marfan syndrome [European Society of Gynecology et al., 2011]. Nevertheless, our report shows that a dedicated cardiac screening with a fetal echocardiography may be of interest to these women and to women whose partner has Marfan syndrome. Finally, genetic counseling remains of high interest for patients with genetically documented Marfan syndrome, regardless of the phenotype severity. This should always be proposed to patients, who will finally make the ultimate decision to continue with or refuse further investigations.

\section{Acknowledgment}

The authors thank the patients for their participation in this study.

\section{Statement of Ethics}

The research was ethically conducted in accordance with the World Medical Association Declaration of Helsinki. The patient has given informed written consent.

\section{Disclosure Statement}

The authors have no disclosure to declare.

\section{References}

Atypical Familial Marfan Syndrome
- Bolar N, Van Laer L, Loeys BL: Marfan syndrome: from gene to therapy. Curr Opin Pediatr 24: 498-504 (2012).

Booms P, Cisler J, Mathews KR, Godfrey M, Tiecke F, et al: Novel exon skipping mutation in the fibrillin-1 gene: two 'hot spots' for the neonatal Marfan syndrome. Clin Genet 55: 110-117 (1999).

- Brito-Filho SL, Oporto V, Campos O, Alvares AB, Carvalho AC: A case of neonatal Marfan syndrome with good late follow-up: is it possible to avoid an early unfavourable outcome? Cardiol Young 23:301-303 (2013).

-Collod-Béroud G, Lackmy-Port-Lys M, Jondeau G, Mathieu M, Maingourd Y, et al: Demonstration of the recurrence of Marfan-like skeletal and cardiovascular manifestations due to germline mosaicism for an FBN1 mutation. Am J Hum Genet 65:917-921 (1999).

Elshershari H, Harris C: Paternal fibrillin-1 mutation transmitted to an affected son with neonatal Marfan syndrome: the importance of early recognition. Cardiol Young 24:735-738 (2013).

European Society of Gynecology (ESG); Association for European Paediatric Cardiology (AEPC); German Society for Gender Medicine (DGesGM), Regitz-Zagrosek V, Blomstrom Lundqvist C, et al: ESC Guidelines on the management of cardiovascular diseases during pregnancy: the Task Force on the Management of Cardiovascular Diseases during Pregnancy of the European Society of Cardiology (ESC). Eur Heart J 32:3147-3197 (2011). 
Faivre L, Collod-Beroud G, Callewaert B, Child A, Binquet $\mathrm{C}$, et al: Clinical and mutation-type analysis from an international series of 198 probands with a pathogenic FBN1 exons 2432 mutation. Eur J Hum Genet 17:491-501 (2009a).

-Faivre L, Masurel-Paulet A, Collod-Béroud G, Callewaert BL, Child AH, et al: Clinical and molecular study of 320 children with Marfan syndrome and related type I fibrillinopathies in a series of 1,009 probands with pathogenic FBN1 mutations. Pediatrics 123:391-398 (2009b).

- Gavilán C, Herraiz I, Granados MA, Moral MT, Gómez-Montes E, Galindo A: Prenatal diagnosis of neonatal Marfan syndrome. Prenat Diagn 31:610-613 (2011).

-Hennekam RC: Severe infantile Marfan syndrome versus neonatal Marfan syndrome. Am J Med Genet A 139:1 (2005).

-Hilhorst-Hofstee Y, Hamel BC, Verheij JB, Rijlaarsdam ME, Mancini GM, et al: The clinical spectrum of complete FBN1 allele deletions. Eur J Hum Genet 19:247-252 (2011).

Montgomery RA, Geraghty MT, Bull E, Gelb BD, Johnson M, et al: Multiple molecular mechanisms underlying subdiagnostic variants of Marfan syndrome. Am J Hum Genet 63: 1703-1711 (1998).
Morse RP, Rockenmacher S, Pyeritz RE, Sanders SP, Bieber FR, et al: Diagnosis and management of infantile Marfan syndrome. Pediatrics 86:888-895 (1990).

Putnam EA, Cho M, Zinn AB, Towbin JA, Byers $\mathrm{PH}$, Milewicz DM: Delineation of the Marfan phenotype associated with mutations in exons 23-32 of the FBN1 gene. Am J Med Genet 62:233-242 (1996).

Rantamäki T, Kaitila I, Syvänen AC, Lukka M, Peltonen L: Recurrence of Marfan syndrome as a result of parental germ-line mosaicism for an FBN1 mutation. Am J Hum Genet 64:9931001 (1999).

Rozendaal L, Blom NA, Hilhorst-Hofstee Y, Ten Harkel AD: Dilatation of the great arteries in an infant with Marfan syndrome and ventricular septal defect. Case Rep Med 2011:172109 (2011).

-Sipek A, Grodecká L, Baxová A, Cibulková P, Dvořáková $\mathrm{M}$, et al: Novel FBN1 gene mutation and maternal germinal mosaicism as the cause of neonatal form of Marfan syndrome. Am J Med Genet A 164A:1559-1564 (2014).
Stheneur C, Faivre L, Collod-Béroud G, Gautier $\mathrm{E}$, Binquet $\mathrm{C}$, et al: Prognosis factors in probands with an FBN1 mutation diagnosed before the age of 1 year. Pediatr Res 69:265-270 (2011).

Sutherell J, Zarate Y, Tinkle BT, Markham LW, Cripe LH, et al: Novel fibrillin 1 mutation in a case of neonatal Marfan syndrome: the increasing importance of early recognition. Congenit Heart Dis 2:342-346 (2007).

Tekin M, Cengiz FB, Ayberkin E, Kendirli T, Fitoz $S$, et al: Familial neonatal Marfan syndrome due to parental mosaicism of a missense mutation in the FBN1 gene. Am J Med Genet A 143A:875-880 (2007).

Tiecke F, Katzke S, Booms P, Robinson PN, Neumann L, et al: Classic, atypically severe and neonatal Marfan syndrome: twelve mutations and genotype-phenotype correlations in FBN1 exons 24-40. Eur J Hum Genet 9:13-21 (2001).

Wang M, Kishnani P, Decker-Phillips M, Kahler SG, Chen YT, Godfrey M: Double mutant fibrillin-1 (FBN1) allele in a patient with neonatal Marfan syndrome. J Med Genet 33:760763 (1996). 